\title{
Real Time Sticky Bomb Detection System Based on Compass Device and Arduino Board
}

\author{
Sameer Hameed Majeed \\ Computer Technology Eng. Dep. \\ Al-Mansour University College \\ Baghdad, Iraq \\ samer1607@gmail.com
}

\author{
Noor Kareem Jumaa \\ Computer Technology Eng. Dep. \\ Al-Mansour University College \\ Baghdad, Iraq \\ Noor.k88@gmail.com
}

\author{
Auday A.H. Mohamad \\ Computer Technology Eng. Dep. \\ Al-Mansour University College \\ Baghdad, Iraq \\ auday_abdulhussein@muc.edu.iq
}

\begin{abstract}
This paper presents a new strategy of sticky bomb detection. The detection strategy is based on measuring the magnetic field around the targeted car using compass device. A compass measure the earth gravitation of the car as $(x, y, z)$ coordination, a threshold value of magnetic fields around the targeted car are recorded. If a difference is detected with any $(x, y, z)$ coordination, an alert SMS message is sent to the car's owner. The detection system presented in this paper has been implemented based on Arduino board. The alarm signal is a Short Message Service (SMS) through Global System for Mobile Communication (GSM) module. The proposed method can gives the people of unstable countries a chance to discover whether their cars have been trapped with an IED bomb or their car still safe.
\end{abstract}

Index Terms-compass, Mass spectroscopy, Magnetic analysis, Microsensors, Magnetic sensors

\section{INTRODUCTION}

In present days, there is lot of attention to the development of techniques and devices for explosives detection. Most of explosives attacks are done with magnetic sticky bombs trapping cars of other peoples. Explosive attacks become more hazardous since the bombs can be launched using remote control by using of mobile phones which allows starting a bomb immediately. Therefore, an early alarm is needed to warn the car owner from possible Improvised Explosive Devices (IED) in his ther car.

Anti-social elements are used IEDs for making terror attacks remotely using mobile phones and other wireless devices, these explosives lead to several thousands of people lost their lives and even more number of people injured. Since government infrastructures and people are targets to explosive attacks, reliable detection systems to protect people and possessions from dangerous attacks are an essential need [1].

Recently some attempts were found in the literature to deploy sensors to form wireless sensor network that can detect IEDs and some other approaches for IED bombs detection. There is much room for research into the area of detecting explosives caused by IEDs $[1,2]$.

Many principles are studied to detect explosives caused by IEDs. The spectroscopic principle method is used in the bomb detection system in this paper. Mass spectroscopic principle method based on electromagnetic field around the targeted car has been used. The electromagnetic field of the car is a result of the earth gravitation and a rounded disturbance. According to the change of the electromagnetic field due to strange mass attached to the car; the proposed system detects the possibility to be an IED bomb.

The paper is arranged in five sections. section 2 presents a survey of related works, section 3 discuss the improvised explosive devices (IED) detection methods, section 4 describe the details of the proposed system, and section 5 discuss the conclusions of this work.

\section{RELATED WORKS}

In [1], an explosive detection system is surveyed based on wireless sensor network (WSN), and the researchers intend to simulate the system. The researchers threw light into different aspects of explosives, their characterization, detection and possible technologies for counter measures. In 
[2], an explosive detection system is proposed which automatically detects the IED without any human intervention. Sensors and inbuilt communication system are coated with chemical compounds that are frequently used in IEDs.

In [3], a method was proposed to detect magnetic sticky bomb, the researchers obtained a result for parked car. A compass and magnetometer are used to obtained the results of the magnetic measurements which were listed in table(1) of their research.

A discussion on explosive detection is presented in [4], the researcher discuss the design of single chip nanonose is a tedious job and requires a very accurate alignment of nanosensors and appropriate interfacing parameters.

A detector consists of multiple ultrasonic transducers in all directions within the specified boundary allotted combined to form a neutral network to locate the hotspot by using the DASH7 technology that can be integrated with PC has been proposed in [5]. In [6], actually applied methods of detecting vapors of explosives have been presented. They present methods which are characterized by a high degree of technological development and magnetic resonance devices were analyzed and the magnetic detection method was explained in page 19 of the research.

\section{IMPROVISED EXPLOSIVE DEVICES (IED) DETECTION}

IEDs are listed as a new bomb attacks which are more sophisticated, more dangerous, terrorists use mobile phone to initiate a bomb immediately. An IED is an unpremeditated explosive charge, equipped with a home-made (i.e. non-standard) or a professional detonator. Improvised Explosive (IE) could be any chemical or a mixture of capable explosive reaction. Typically, IE components are inorganic salts containing molecular bound oxygen, like nitrates, chlorates, perchlorates or organic mixtures with nitro-, nit amine- or nitrate-groups or peroxIEDs [2, 6 and 7].

Typically, IED detection techniques can be divided into two groups: bulk detection of explosives, and trace detection of explosives [6].
In bulk detection, a macroscopic mass approach is applied, in which macroscopic mass of explosive material is directly detected, by viewing images made by X-ray scanners or similar equipment [6].

In trace detection, the explosive detection is based on chemical Identification of microscopic residues of the explosive compound. There are two forms for applying these residues, which can be applied in either or both of two forms: vapor and particulate. The vapor detection is referring to gas-phase molecules that emitted by a solid or liquid explosives. The explosives concentration in the air is associated to the vapor pressure of the explosive material and to other factors, for example: the duration of materials of the presence explosive in the given location, its packing, temperature, circulation of air in the location, etc. In particulate detection, microscopic particles of solid explosive material are stand by to the surface by the contacting directly with the explosive, or indirectly, by it's contacting with hands of someone in handling the explosives [5, 6]. Vapor detectors search for very small quantities, less than a microgram unlike bulk detection which is aimed to detect large quantities of explosives. Hydrogen, Nitrogen, Carbon and Oxygen are the main components in an explosive [6].

\section{Proposed System}

The aim of this paper is to use one of several detection ways to prevent a vehicle from being attacked by sticking a bomb on the body while its owner is a way from it. This system is implemented using low cost electronic items available in the local market.

The proposed system is designed according to the mass spectroscopic approach. In this system, the IED detection system is composed of Arduino board, GSM module, and a compass device. The role of Arduino is to drive the compass which is used for the detection of IED. The compass sensor is used to measure the electromagnetic field around the car frequently; the car has an initial electromagnetic field caused by the gravitation of the earth and other sources of electromagnetic fields, if a change occurs in electromagnetic field around the car, the Arduino 
board will initiate a SMS message based on the GSM module to the car owner. The proposed method can gives the people of unstable countries a chance to discover whether their cars have been trapped with an IED bomb or their car still safe.

\section{A) System Block Diagram}

The proposed explosive detection system architecture consists of Arduino Uno board, GSM module, and a compass device. System structure is shown in Fig.1.

The GSM board is connected to the Arduino board by pins $(7,8)$, the usage of these pins is to send and receive data serially between Arduino board and GSM shield. The HMC (compass) shield is connected to the board through pin (A4, A5) to communicate with board via $\mathrm{I}^{2} \mathrm{C}$ protocol.

\section{B) System Components}

This subsection explains the structure and operation of modules used in the proposed system architecture.

\section{Arduino Uno Board [8]}

Arduino is an open-source computer hardware and software company, the user community that designs and manufactures kits for building digital devices can be used to control the physical world. There are different types of Arduino board such as Arduino Uno, Arduino NG, Decimilia, Duemilanove, Arduino Mega 2560, Arduino Mega ADK, and Arduino LilyPad.

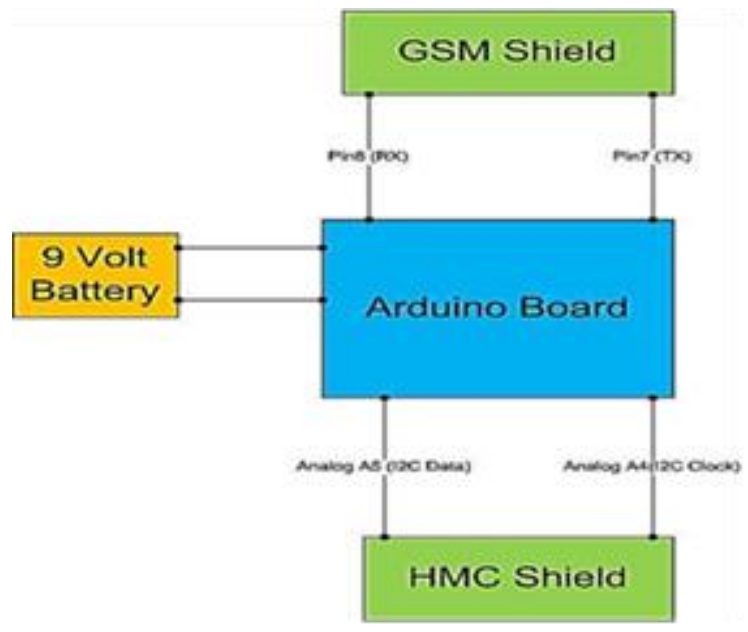

Fig.1 Proposed System Block Diagram
In this paper, Arduino Uno R3 board (shown in Fig. 2) has been used, which is a microcontroller board based on the ATmega328. It has 14 digital I/O pins (of which 6 can be used as PWM outputs), 6 analog inputs, a $16 \mathrm{MHz}$ crystal oscillator, a USB connection, a power jack, and a rest button. It contains everything needed to support the microcontroller; simply connect it to a computer with a USB cable or power it with an AC-to-DC adapter or battery to get started.

Arduino board contains several types of serial communication, USART, $\mathrm{I}^{2} \mathrm{C}$, and SPI. In this paper, the board will communicate with compass through $\mathrm{I}^{2} \mathrm{C}$ protocol and will communicate with GSM board through UART protocol.

2. GSM Module (SIM900 GSM Cellular Shield) [9]

The SIM900 GSM/GPRS Shield provides a way to use the GSM cell phone network to receive data from a remote location. The shield allows achieving this via any of the three methods:

- Short Message Service

- Phone Call Service

- GPRS Service

The GPRS Shield is compatible with all boards which have the same from factor (and pinout) as a standard Arduino Board. The GPRS Shield is configured and controlled via its USART using simple AT commands. Based on the SIM900 module from SIMCOM Company, the GPRS Shield is like a cell phone. Fig. 3 shows a sample of this shield.

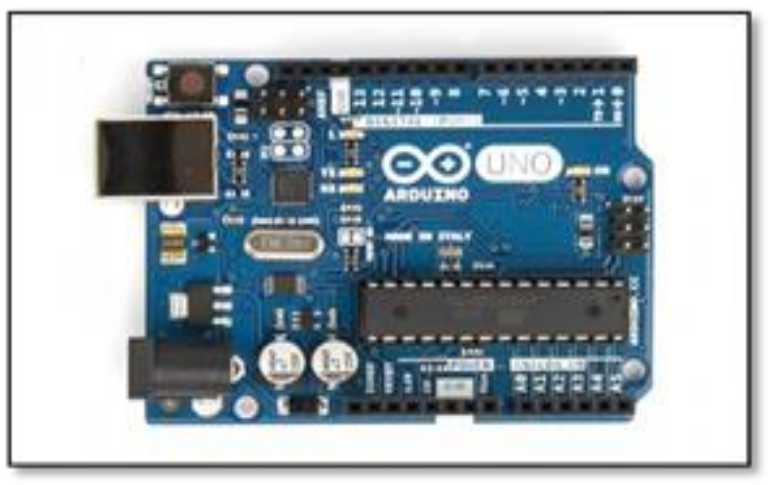

Fig.2 Arduino UNO board 
3. Compass (Digital Compass (HMC5883L)) [10]

This component allows the user to measure both the direction and the magnitude of the Earth's magnetic field. The HMC5883L magnetometer has within 3 magneto-resistive sensors arranged on three perpendicular axes (the Cartesian axes $\mathrm{x}$, y, and z). Fig. 4 shows HMC5883L.

The direction of Earth's magnetic fields affects the flow of electrons in the sensor, and those changes in current can be measured and calculated to drive a compass heading or other useful information. The component HMC5883L can communicates with Arduino through the $\mathrm{I}^{2} \mathrm{C}$ protocol. The connection diagram of HMC5883L with Arduino-Uno board is shown in Fig. (5).

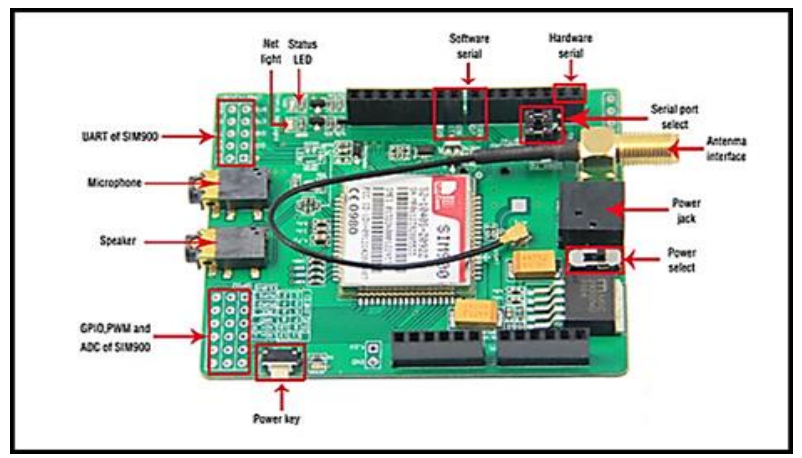

Fig.3 SIM Card/GPRS Shield.

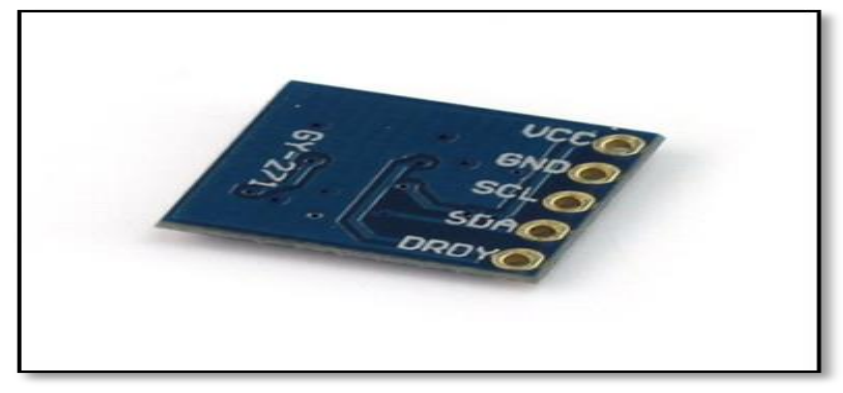

Fig.4 HMC5883L Digital Compass

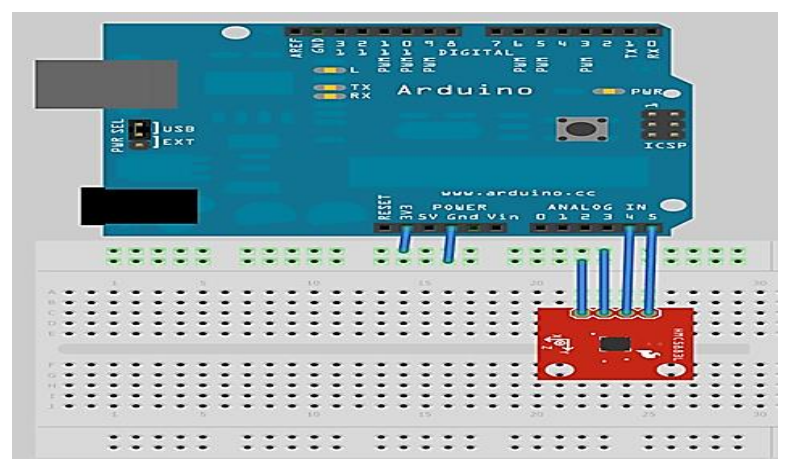

Fig.5 HMC5883L Connection Diagram with Arduino Board.
4. System Operation, Software and Results

The operations of this system can be demonstrated with flowchart of Fig. 6. The first part of the code is to define the $\mathrm{I}^{2} \mathrm{C}$, software serial, HMC and GSM libraries to the board to enable the board communicate with these shields by sending or receiving data as shown:

\#include 〈Wire.h>

\#include <String.h>

\#include <SoftwareSerial.h>

SoftwareSerial SIM900(7, 8);

\#include <I2Cdev.h>

\#include <HMC5883L.h>

HMC5883L mag;

Some variables must be defined to store the electromagnetic field values that are sent by the HMC object ( $\mathrm{x}$ axis, $\mathrm{y}$ axis and $\mathrm{z}$ axis):

int16_t mx, $m y, m z, x, y, z$;

Below commands are used to let the I2C communication start working:

void setup ( ) \{

Wire.begin( );

Serial.println("Initializing I2C devices..."); mag.initialize ();

Below commands that are used to start serial UART communication between Arduino and GSM board:

Serial.begin(19200);

SIM900.begin(19200);

// verify connection

Serial.println("Testing device connections...");

Serial.println(mag.testConnection( ) ? "HMC5883L connection successful": "HMC5883L connection failed");

Below commands are written to read the initial values of the HMC object and store the (x,y and $\mathrm{z}$ axis), these values will be stored in the memory of the microcontroller and compare them with all followed values if there's a difference in between one of these values with the new one then there's 
a problem with the electromagnetic filed around the car.

mag.getHeading $(\& x, \& y, \& z)$;

Serial.println("'"');

Serial.print(x); Serial.print(" $\left.\backslash t^{\prime \prime}\right)$;

Serial.print(y); Serial.print(" $\left.\mid t^{\prime \prime}\right)$;

Serial.print(z); Serial.print(' $\left.\backslash t^{\prime \prime}\right)$;

Serial.println("'"');

Serial.println("'"');

Below are commands used to monitor the field around the car and keep compare the values with the initial values, if there's a difference between one of them then an action will be taken to warn the car owner such as sending Short message or start an alarm system in the car.

void loop( ) \{

mag.getHeading(\&mx, \&my, \&mz);

$(m x>x+10$ or $m x<x-10$ or $m y>y+10$ or $m y<y-10$ or $m z>z+10$ or $m z<z-10)$

$\{\operatorname{SendSMS(~);~}$

ShowSerialData( );

while(1) \{\}

\}

else\{

Serial.print("mag: $\left.t^{\prime \prime}\right)$;

Serial.print(mx); Serial.print(' $\left.\backslash t^{\prime \prime}\right)$;

Serial.print(my); Serial.print(' $\left.\backslash t^{\prime \prime}\right)$;

Serial.print(mz); Serial.print( $\left."{ }^{\prime \prime} t^{\prime \prime}\right)$;

Serial.println();\}

$\operatorname{delay}(5000) ;\}$

Below is the part of code which is used to enable let the Arduino board send short message to the car owner to warn him/her there's a difference in the field around the car.

void SendSMS( )\{

SIM900.print("AT+CMGF=1 ${ }^{\prime \prime}$ ");

$\operatorname{delay}(1000)$;
SIM900.print("AT+CMGS $=l^{\prime \prime}+\operatorname{xxx\times x\times xxxx|^{\prime \prime }\vee }$

');

delay(1000);

SIM900.println("Check your vehicle, there's something attached");

SIM900.print( ' $\left.\backslash r^{\prime \prime}\right)$;

delay (1000);

SIM900.println((char)26);

SIM900.println( );\}

void ShowSerialData( )\{

while(SIM900.available( )!=0)

Serial.write(SIM900.read( ));\}

Fig.7 shows the initial values of magnetic fields in three axes. These values are the test results of the proposed system without any external sources of magnetic fields. The first column of these readings is represents the value of magnetic field in x-axis, the second column is represents the value of magnetic field in y-axis while the third column represents the value of magnetic field in $\mathrm{z}$-axis. These readings are recorded without any external source of magnetic field (Permanent Magnet which is used in sticky bomb). There is a small fluctuation in readings with time (the time between two readings is set to five seconds). So, these readings can be regards as the threshold values of current situation of the protected car (the threshold is initiated and recorded when the car is stopped in a certain place and this value can be change from place to another).

Fig. 8 shows the values of magnetic field in three axes when an external source of magnetic field (permanent magnet) is installed near the compass sensor which is represents a sticky bomb. There is difference in readings can be noted, so an SMS message can be initiated to the car's owner. 


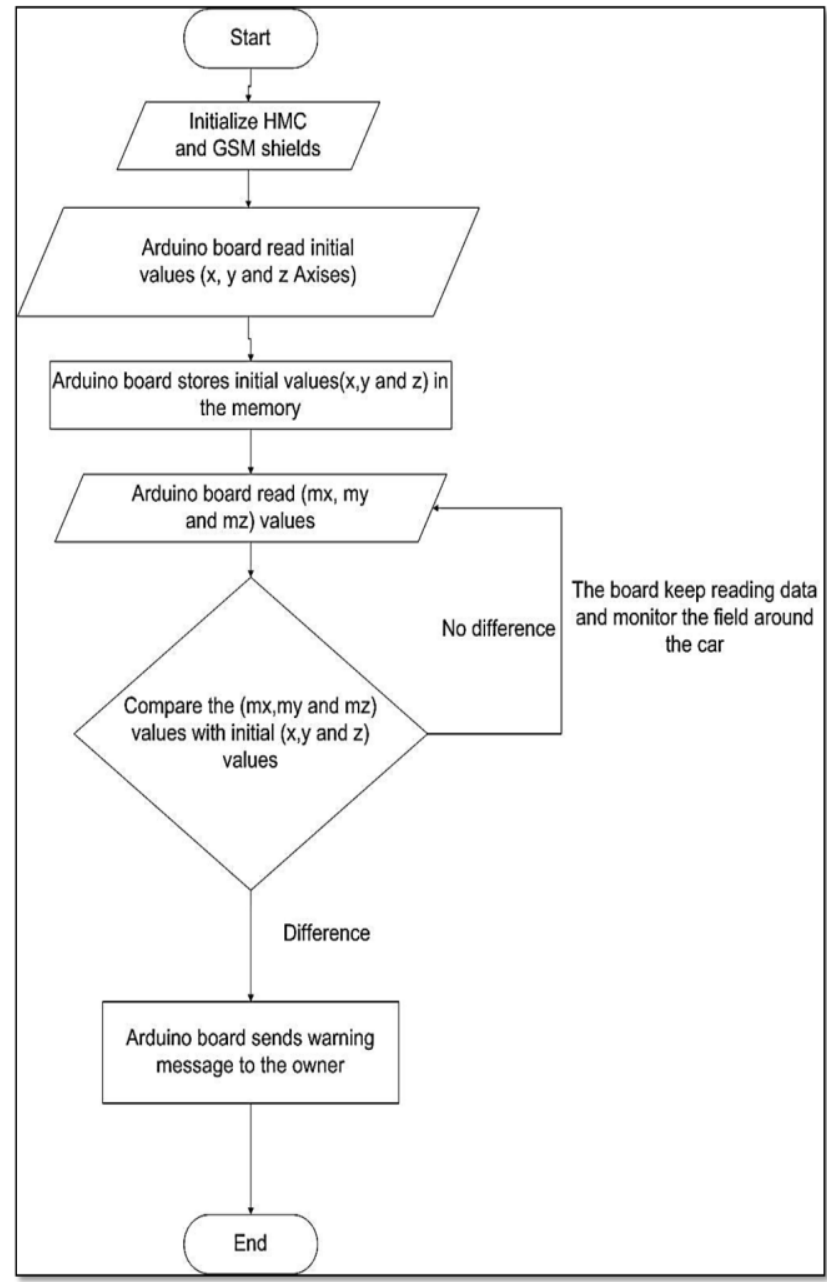

Fig.6 Proposed System Flowchart

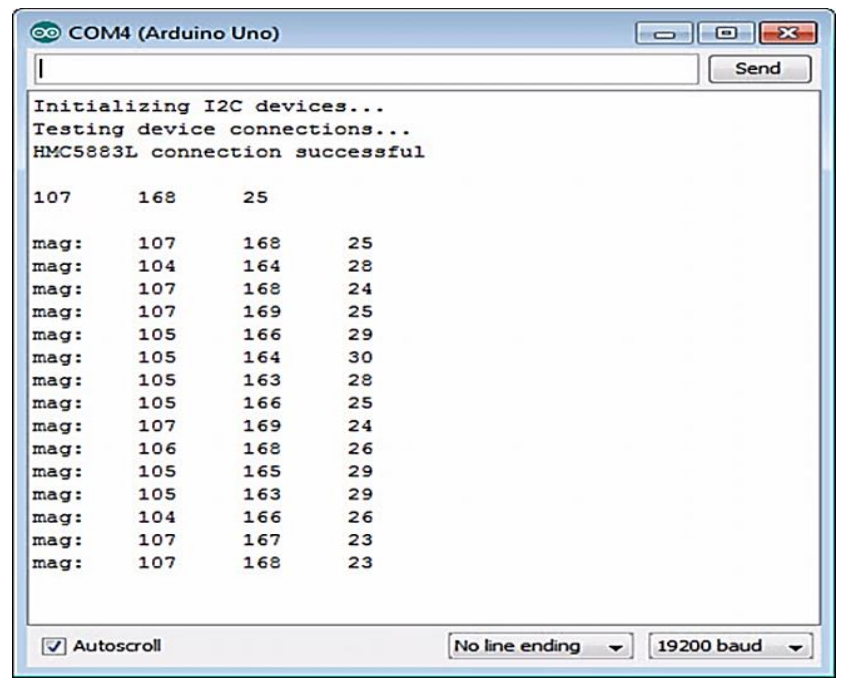

Fig.7 Initial Values of Magnetic Fields in Three Axes

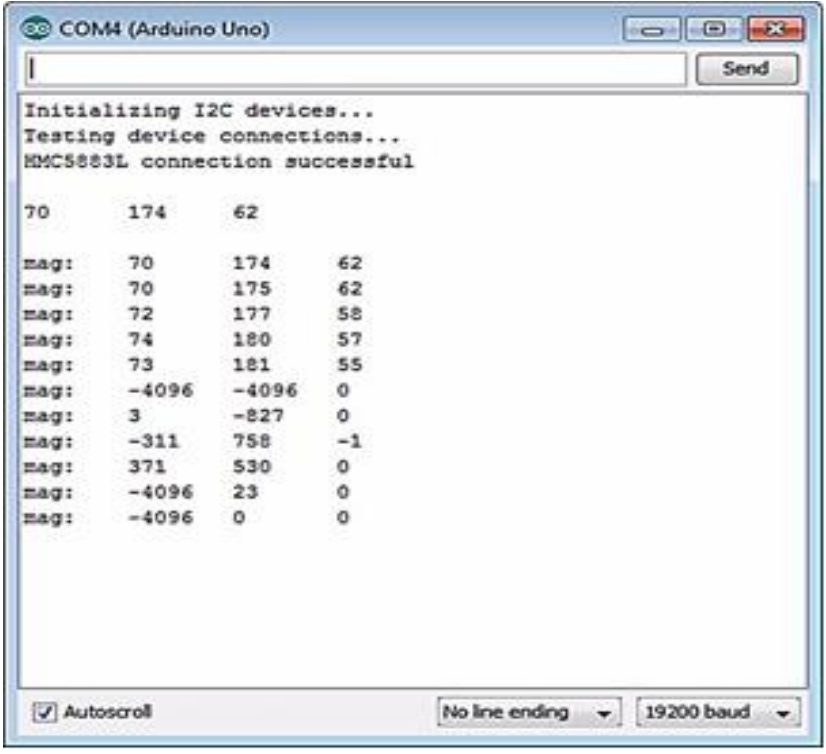

Fig.8 Magnetic Field in three axes with an External Source.

The following figures showing a real time test of the proposed system. Fig. 9a shows the initial magnetic field of the car, while Fig. 9b shows the magnetic field of the car after adding a magnate which perform a sticky magnetic bomb ( the placement of sticky magnetic bomb does not effect on the operation of the system because the value of magnetic field of permanent magnet is large enough to make a small difference with the initial value), a warning message then will be sent to the car owner phone; warning message text is " Check your vehicle, there's something attached".

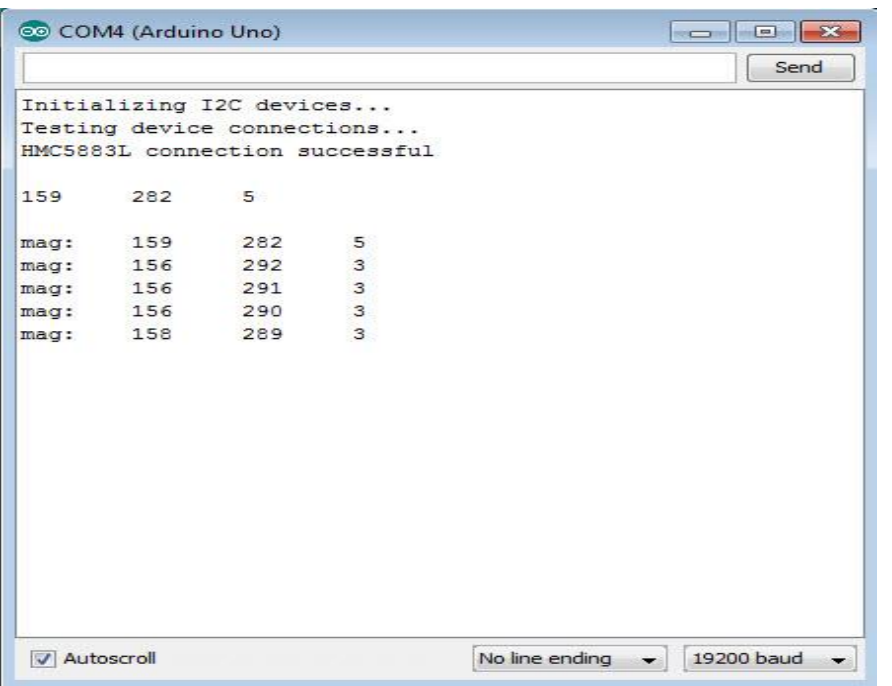

Fig.9a Initial Values of Magnetic Fields of the Car 


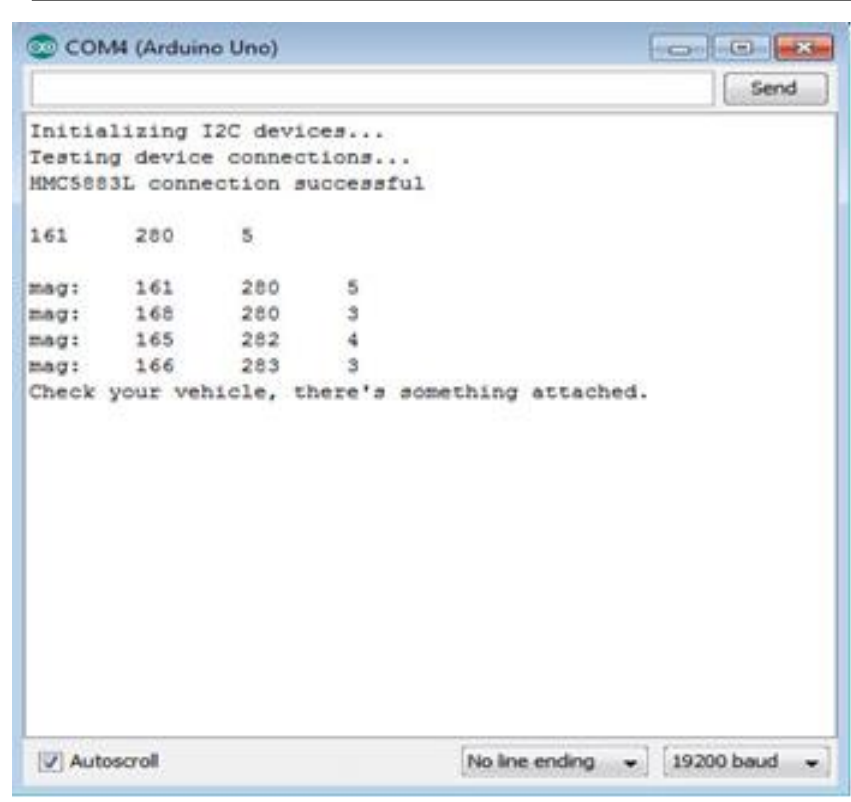

Fig.9b Magnetic Field after adding magnate to the car and the warning message.

An important note should be mentioned, each car has a magnetic field which is differ from the magnetic field of other cars since each car has its weight, mass, wheel size, and other properties that makes the threshold of magnetic field fixed to each car.

\section{Conclusions}

The automatic explosive detection system proposed in this research is detecting the IED automatically without any intervention of humans. The proposed system has many advantages when compared with the traditional detection techniques in which the proposed system has less cost, low power consumption and it take less analysis time than other traditional detection systems. The proposed system can sent the exact location of the car that possible to contain an IED which can deactivated immediately so that many people can be saved from a possible explosive.

\section{REFERENCES}

[1] R V Kishore Kumar and Dr. G.Murali, "A Survey on the Present State-of-the-Art of Explosives, Detection Methodsand Automatic Explosive Detection using Wireless Sensor Network", International Journal of Applied Engineering Research, ISSN 0973-4562, Volume 11, Number 1, pp. 504-510, 2016.
[2] Journal of Applied Engineering Research, ISSN 0973-4562, Volume 11, Number 1, pp. 504-510, 2016.

[3] Avinash.Vanimireddy and D.Aruna Kumari, "Detection of Explosives Using Wireless Sensor Networks", International Journal of Engineering Trends and Technology, Volume3, 2012.

[4] Roger G. Johnston, Jim Vetrone, and John S. Warner, "Sticky Bomb Detection with Other Implications for Vehicle Security", Journal of Physical Security 4(1), PP 36-46, 2010.

[5] S. A. Desai, P. H. Zope, and S. R. Suralkar, "A Discussion on Explosive Detection", International Journal of Advanced Research in Electrical, Electronics and Instrumentation Engineering Vol. 1, ISSN: 2278 - 8875, 2012.

[6] M.B.Muthukumaresan, V.S.Ramesh, and S.Jagadishkumar, "gED - A Quantitative Approach To Detect Explosives", International Journal of Engineering and Technology, Volume 2, ISSN: 2049-3444, No. 7, 2012.

[7] Zbigniew Bielecki, Jacek Janucki, Adam Kawalec, Janusz Mikołajczyk, Norbert Pałka, Mateusz Pasternak, Tadeusz Pustelny, Tadeusz Stacewicz, and Jacek Wojtas, "Sensors and Systems for the detection of Explosive Devices-An Overview", Metrology and Measurements Systems, ISSN 0860-8229, Vol. XIX (2012), No. 1, pp. 328.

[8] Mradul Jain, Puneet Goyal, and Anmol Jain, " Early Warning System for Improvised Explosive Detection Using Wireless Sensor Networks: An Intelligent Information Directed Approach", International Journal of Advanced Research in Computer Science and Software Engineering, ISSN: 2277 128X, Volume 6, 2016.

[9] www.arduino.cc.

[10] Advance Technical Research And Innovation For Mankind (ATRIM) Electronics, "GSM Shield Manual", 2014, (C)ATRIM Electronics Pvt Ltd|www.atrim.in.

[11] HMC5883LManual, www.magneticsensors .com, (C2010 Honeywell International Inc. 